\title{
五个天然异戊烯基黄酮的首次全合成研究
}

\author{
冯尧 杨金会* 马晓琴 陈兵兵 谢一民 \\ (宁夏大学天然气转化国家重点实验室培育基地 银川 750021)
}

\begin{abstract}
摘要 完成了具有 2,3-二羟基-3-甲基丁基黄酮化合物 brosimacutin A-B, brosimacutin D, brosimacutin E, brosimacutin H 和 brosimacutin M 五个黄酮类化合物的消旋体的合成. 所有新化合物的结构都经过 NMR, HRMS 确认.

关键词 异戊烯基黄酮; 查尔酮; 二氢查尔酮; 全合成; brosimacutin
\end{abstract}

\section{First Total Synthesis of Five Natural Prenylated Flavonoids}

\author{
Feng, Yao Yang, Jinhui* Ma, Xiaoqin Chen, Bingbing Xie, Yimin \\ (State Key Laboratory Cultivation Base of Natural Gas Conversion, Ningxia University, Yinchuan 750021)
}

\begin{abstract}
A simple and efficient approach for synthesis flavonoids with 2,3-dihydroxy-3-methylbutyl moiety, by which seven natural prenylated flavonoids, brosimacutin $\mathrm{A}-\mathrm{B}$, brosimacutin $\mathrm{D}$, brosimacutin $\mathrm{E}$, brosimacutin $\mathrm{H}$ and brosimacutin $\mathrm{M}$ have been synthesized in racemic form. All structures of new compounds were confirmed by NMR and HRMS.

Keywords prenylated flavonoids; chalcone; dihydrochalcones; total synthetic; brosimacutin
\end{abstract}

天然异戊烯基黄酮广泛地存在于自然界中，并且具 有广泛的生理及药理活性, 如抗氧化 ${ }^{[1]}$ 、抗癌 ${ }^{[2]}$ 、抗病 毒 ${ }^{[3,4]}$ 等. 异戊烯基的位置以及数量会影响黄酮类化合 物的一些活性 ${ }^{[5]}$. 异戊烯基可以给黄酮化合物提供结构 以及数量的多样性, 如对异成烯基侧链的氧化还原, 羟 基化, 环化成五、六元环等 ${ }^{[6]}$. 对于含有异戊烯基侧链的 黄酮类天然产物, 缺乏一种有效的合成方法. 对于该类 化合物的合成方法将有利于相关生物活性与结构关系 的进一步研究.

Brosimacutins 1 5(图 1)是从 brosimum acutifolium huber (Moraceae) ${ }^{[7,8]}$ 的根部中分离出的 5 个黄酮类化合 物. 这种植物的根部在当地被广泛地用于抗抗炎和抗风 湿病. 据 Takashima 等 ${ }^{[9]}$ 报道包括化合物 $\mathbf{1} \sim \mathbf{5}$ 在内的 27 个黄酮化合物对 mouse leukemia P388 细胞具有一定的 细胞活性. 尽管 brosimacutins 1 5 在很多年以前就被 分离, 但是对于其的合成还未见报道.

基于本课题组在这一领域的研究 ${ }^{[10 ~ 13]}$, 我们报道 了化合物 $\mathbf{1} \sim \mathbf{5}$ 的合成(Scheme 1).

\section{1 结果与讨论}

化合物 $1 \sim 5$ 具有相似的结构. 我们可以认为化合 物 10 是 $1 \sim 5$ 的生物学前体. 合成化合物 $1 \sim 5$ 的关键是: 怎样、何时构建二美基结构以及相关的官能团转化(图 2).

我们由 2,4-二羟基苯乙酮(6)和对羟基苯甲醛为起 始原料, 其羟基由芐基保护. 化合物 7 和 4-芐氧基苯甲 醛在 $\mathrm{KOH} /$ 乙醇的条件下羟醛缩合以 $87 \%$ 的产率生成查 尔酮 8 和少量的黄烷酮 9 . 随后查尔酮 8 分子内迈克尔 加成以 $36 \%$ 的产率得到了黄烷酮 9. 需要指出的是加入 催化量的 18-冠-6 后, 产率提高到了 $67 \%{ }^{[14]}$.

化合物 9 在间氯过氧苯甲酸 $(m-\mathrm{CPBA})$ 的条件下得 到了环氧化合物 10. 在不同的条件下环氧与选择性环 化得到了 brosimacutin D 和 brosimacutin E (5). 化合物 10 以 1,4-cyclohexadiene 提供氢源, 在 $\mathrm{Pd} / \mathrm{CaCO}_{3}$ 的条件 下以 $83 \%$ 的产率得到了苯并呋喃 brosimacutin $\mathrm{E}(\mathbf{5})^{[15]}$.

brosimacutin D (4)由两步反应得到: (1) Pd-C 在 $\mathrm{H}_{2}$ 氛围下脱去芐基; (2)用 $\mathrm{HCOOH}$ 环化 ${ }^{[16]}$. 因为 $4^{\prime}-\mathrm{OH}$ 的

\footnotetext{
*E-mail: yang_jh@nxu.edu.cn

Received June 14, 2014; revised August 8, 2014; published online August 26, 2014.

Project supported by the National Natural Science Foundation of China (Nos. 21162021, 21362025) and the Program for New Century Excellent Talents in University (No. NCET-09-0860).

国家自然科学基金(Nos. 21162021, 21362025)、教育部新世纪优秀人才支持计划(No. NCET-09-0860)资助项目.
} 
<smiles>CC(C)(O)C(O)Cc1c(O)ccc2c1OC(c1ccc(O)cc1)CC2=O</smiles>

Brosimacutin A-B

(1)<smiles>CC(C)(O)C(O)Cc1c(O)ccc(C(=O)CCc2ccc(O)cc2)c1O</smiles>

(3)<smiles>CC(C)(O)C(O)Cc1c(O)ccc(C(=O)/C=C/c2ccc(O)cc2)c1O</smiles>

Brosimacutin M

(2)<smiles>CC1(C)Oc2ccc3c(c2CC1O)O[C@H](c1ccc(O)cc1)CC3=O</smiles>

(4)<smiles>CC(C)(O)C1Cc2c(ccc3c2O[C@@H](c2ccc(O)cc2)CC3=O)O1</smiles>

(5)

图 1 化合物 $\mathbf{1} \sim \mathbf{5}$ 的结构

Figure 1 Structures of compounds $\mathbf{1} \sim \mathbf{5}$

活性较低, 因此目标产物 4 以相对较低的产率得到
(Scheme 2).

化合物 $1 \sim 3$ 具有相同的侧链基团: 2,3-二差基-3-甲 基丁基. 这可以由异戊烯基得到. 有几种异戊烯基二羟 基化的方法如: $\mathrm{KMnO}_{4}, \mathrm{OsO}_{4}{ }^{[17]}$, oxone ${ }^{[18]}$, enzyme ${ }^{[5]}$ 等. $\mathrm{KMnO}_{4}$ 需要在碱性的条件下, 这可能导致 C-环的开环. $\mathrm{OsO}_{4}$ 毒性较大, 且价格较为昂贵, 我们将其排除. 据报 道 oxone 可以将双键转化为二醇, 但是我们尝试用 oxone 氧化化合物 9, 没有得到化合物 11, 只得到了环 氧化合物 10 且产率较低(Scheme 3). 最后我们采用了一 个两步的策略：用 $m$-CPBA 氧化 9 以 $68 \%$ 的产率得到了 环氧化合物 10 , 随后在 $\mathrm{H}_{2} \mathrm{SO}_{4}(49 \%)$ 的条件下以 $74 \%$ 的 产率得到了二醇化合物 $\mathbf{1 1}^{[19]}$. 值得指出的是在这种条 件下 C-环未开环. 这种方法相对的温和、环保，对于黄 酮类化合物的合成尤为合适.

化合物 11 以 Pd-C 催化加氢脱去苠基以 $85 \%$ 的产率 的到了 brosimacutins (1). Brosimacutins A-B (1)在 4 $\mathrm{mol} / \mathrm{L} \mathrm{NaOH}$ 的条件下开环以 $77 \%$ 的产率得到查尔酮 brosimacutin $\mathrm{M}(\mathbf{2})^{[20]}$, 因为 brosimacutins A-B (1) 具有较 好的水溶性，因此反应结束后直接乙酸乙酯萃取得到 brosimacutin $\mathrm{M}(2)$ 的纯品. $\mathrm{Pd}-\mathrm{C} / \mathrm{H}_{2}$ 还原查尔醖 brosimacutin M (2)的双键得到二氢查尔酮 brosimacutin H (3).<smiles></smiles>

Brosimacutin D

(4)<smiles>CC(C)(O)CCc1c(O)ccc2c1OC(c1ccc(O)cc1)CC2=O</smiles><smiles>C=C=C</smiles>

Brosimacutin A-B (1)<smiles>CC(O)C1Cc2c(ccc3c2O[C@H](c2ccc(O)cc2)CC3=O)O1</smiles>

(5)<smiles>CC(C)(O)C(O)Cc1c(O)ccc2c1OC(c1ccc(O)cc1)CC2</smiles>

Acutifolin D<smiles>CC(C)(O)C(O)Cc1c(O)ccc(C(=O)/C=C/c2ccc(O)cc2)c1O</smiles>

Brosimacutin M (2)<smiles>C=CCC(=O)c1ccc(O)c(CC(O)C(C)(C)O)c1O</smiles>

(3)

图 2 化合物 $1 \sim 5$ 的反合成分析

Figure 2 Retrosynthetic analysis of compounds $1 \sim 5$ 
<smiles>CC(C)=CCc1c(OCc2ccccc2)ccc(C(=O)SCC(C)C)c1O</smiles><smiles>COc1ccc(C2CC(=O)c3ccc(OCc4ccc(OCc5ccc(O)cc5)cc4)c(c3CC(O)C(C)(C)O)O2)cc1C1CC(=O)c2ccc(O)c(CC(O)C(C)(C)O)c2OC1c1ccc(O)cc1</smiles>

Reagents and conditions: (a) isoprenyl bromide, $\mathrm{KOH}, \mathrm{H}_{2} \mathrm{O}$, r.t., $12 \%$; (b) benzyl chloride, $\mathrm{K}_{2} \mathrm{CO}_{3}, \mathrm{DMF}, 50{ }^{\circ} \mathrm{C}, 61 \%$; (c) $\mathrm{KOH}-\mathrm{H}_{2} \mathrm{O}-\mathrm{EtOH}, \mathrm{N}_{2}, 0$ ${ }^{\circ} \mathrm{C}$ to r.t., $61 \%$; (d) NaOAc, 18 -crown-6, EtOH, reflux, $67 \%$; (e) $m-\mathrm{CPBA}, \mathrm{CH}_{2} \mathrm{Cl}_{2}$, r.t., $68 \%$; (f) $\mathrm{H}_{2} \mathrm{SO}_{4}(49 \%)$, THF, r.t., $71 \%$; (g) $\mathrm{H}_{2}$, $\mathrm{Pd} / \mathrm{C}$, $\mathrm{MeOH}$, EtOAc, r.t., 85\%; (h) $4 \mathrm{~mol} / \mathrm{L} \mathrm{NaOH}$, EtOH, r.t., $77 \%$; (i) $\mathrm{H}_{2}$, $\mathrm{Pd} / \mathrm{C}, \mathrm{MeOH}$, EtOAc, r.t., $50 \%$; (J) (1) Pd-C, EtOAc, $\mathrm{H}_{2}$, r.t., (2) formic acid, r.t., $33 \%$ (2 steps); (k) Pd-black, 1,4-cyclohexadiene, EtOH, $40{ }^{\circ} \mathrm{C}$, r.t., $83 \%$

Scheme 1<smiles>CC(C)(C)Oc1ccc(C2CC(=O)c3ccc(OCc4ccccc4)c(CC4OC4(C)C)c3O2)cc1</smiles>

Scheme 2<smiles>CC(C)=CCc1c(OCc2ccccc2)ccc2c1OC(c1ccc(OCc3ccccc3)cc1)CC2=O</smiles>

\section{2 结论}

本文以 2,4-二羟基苯乙酮和对羟基苯甲醛为起始原 料完成了 brosimacutin A-B, brosimacutin D, brosimacutin $\mathrm{E}$, brosimacutin $\mathrm{H}$ 和 brosimacutin $\mathrm{M}$ 五个黄酮化合物消 旋体的合成. 其波普数据与文献报道一致.

\section{3 实验部分}

\section{1 仪器与试剂}

NMR 用 Bruker (400 and $100 \mathrm{MHz}$ )型核磁共振仪测 定 $\left(\mathrm{CDCl}_{3}, \mathrm{MeOD}\right.$ 或 Acetone- $d_{6}$ 作溶剂, TMS 为内标); HRMS 用 Thermo Orbitrap Elite 测定；200 300 目及 $\mathrm{GF}_{254}$ 硅胶(青岛海洋化工厂生产). 试剂均为分析纯.

Scheme 3 


\section{2 实验方法}

\subsubsection{2-市氧基-3-异戊烯基-4-羟基苯乙酮(7)的合成}

将 3-异戊烯基-2,4-二羟基苯乙酮 (264 mg, 1.2 $\mathrm{mmol})$ 和无水 $\mathrm{K}_{2} \mathrm{CO}_{3}(198.7 \mathrm{mg}, 1.44 \mathrm{mmol})$ 溶于 $10 \mathrm{~mL}$ DMF 中, $65{ }^{\circ} \mathrm{C}$ 下滴加氯化茮 $(182.3 \mathrm{mg}, 1.44 \mathrm{mmol})$, 反 应 $5 \mathrm{~h}$ 后加入少量水, 乙酸乙酯萃取, 合并有机层依次 水洗, 饱和食盐水洗, 无水硫酸钠干燥, 过滤除去干燥 剂, 减压蒸去溶剂, 硅胶柱层析得化合物 7 (226 mg, $0.73 \mathrm{mmol})$, 产率 $61 \%$.

3.2.2 2'-羟基-3'-异戊烯基-4,4'-華氧基查尔酮(8)的合 成

将化合物 7 (561 mg, $1.81 \mathrm{mmol}$ )和 4-芐氧基苯甲醛 $\left(575 \mathrm{mg}, 2.71 \mathrm{mmol}\right.$ )溶于乙醇 $(4 \mathrm{~mL})$ 中, 冷却至 $0{ }^{\circ} \mathrm{C}$, 搅拌下缓慢滴加 $0{ }^{\circ} \mathrm{C}$ 氢氧化钾 $(5.07 \mathrm{~g}, 90.5 \mathrm{mmol})$ 的水 $(4$ $\mathrm{mL})$-乙醇 $\left(8 \mathrm{~mL}\right.$ )溶液, 氮气保护下 $0{ }^{\circ} \mathrm{C}$ 反应 $1 \mathrm{~h}$ 后, 自 然升至室温, 继续室温摚拌反应 $24 \mathrm{~h}$, 将反应物倒入冰 水中, 用 $3 \mathrm{~mol} / \mathrm{L}$ 的盐酸调节至 $\mathrm{pH}<3$, 乙酸乙酯萃取 $(40 \mathrm{~mL} \times 3)$, 合并有机相, 依次水洗, 饱和食盐水洗, 无 水硫酸钠干燥, 减压蒸去溶剂, 硅胶柱层析分离得黄色 油状物 8 (558 mg, $1.11 \mathrm{mmol}, 61.3 \%$ )和化合物 9 (230 $\mathrm{mg}, 0.46 \mathrm{mmol})$, 产率 $25 \% .{ }^{1} \mathrm{H} \mathrm{NMR}\left(400 \mathrm{MHz}, \mathrm{CDCl}_{3}\right)$ $\delta: 13.49$ (s, 1H, OH), 7.85 (d, $J=15.2 \mathrm{~Hz}, 1 \mathrm{H}, \mathrm{H}-\beta), 7.61$ (d, $J=9.2 \mathrm{~Hz}, 1 \mathrm{H}, \operatorname{ArH}), 7.61(\mathrm{~d}, J=8.8 \mathrm{~Hz}, 2 \mathrm{H}, \operatorname{ArH})$ $7.50 \sim 7.31(\mathrm{~m}, 12 \mathrm{H}, \mathrm{ArH}), 7.01(\mathrm{~d}, J=8.4 \mathrm{~Hz}, 2 \mathrm{H}, \mathrm{ArH})$, $6.53(\mathrm{~d}, J=9.2 \mathrm{~Hz}, 1 \mathrm{H}, \mathrm{ArH}), 5.28 \sim 5.24(\mathrm{~m}, 1 \mathrm{H}, \mathrm{CH})$, 5.19 (s, 2H, OCH2), 5.15 (s, 2H, $\left.\mathrm{OCH}_{2}\right), 3.45$ (d, $J=6.8$ $\left.\mathrm{Hz}, 2 \mathrm{H}, \mathrm{CH}_{2}\right), 1.72$ (s, 3H, $\left.\mathrm{CH}_{3}\right), 1.68\left(\mathrm{~s}, 3 \mathrm{H}, \mathrm{CH}_{3}\right) ;{ }^{13} \mathrm{C}$ NMR $\left(100 \mathrm{MHz}, \mathrm{CDCl}_{3}\right) \delta: 192.3,163.2,162.3,160.9$, $144.0,136.6,131.9,130.3,129.0,128.7,128.6,128.3$, $128.1,127.9,127.5,127.2,12.1,118.3,118.0,115.4$, 114.8, 103.3, 70.2, 25.9, 22.0, 17.9; HRMS calcd for $\mathrm{C}_{34} \mathrm{H}_{33} \mathrm{O}_{4}[\mathrm{M}+\mathrm{H}]^{+}:$505.2373, found 505.2368.

\subsubsection{7,4'-二卡氧基-8-异戊烯基黄烷酮(9)的合成}

搅拌下, 将化合物 8 (134 mg, $0.27 \mathrm{mmol}$ ) 溶于 20 $\mathrm{mL}$ 无水乙醇中, 再加入 $(442.8 \mathrm{mg}, 5.4 \mathrm{mmol})$ 无水醋酸 钠和几滴水, 加入少量 18-冠-6, 回流 $25 \mathrm{~h}$, 冷却至室温 向反应物中加少量的水, 用乙酸乙酯萃取 $(20 \mathrm{~mL} \times 3)$, 合并有机相，依次用水、饱和食盐水洗，无水硫酸钠干 燥, 减压蒸去溶剂, 硅胶柱层析分离得淡黄色油状物 9 (89.8 mg, $0.18 \mathrm{mmol}$ ), 产率 67\%. ${ }^{1} \mathrm{H}$ NMR $(400 \mathrm{MHz}$, $\left.\mathrm{CDCl}_{3}\right) \delta: 7.81(\mathrm{~d}, J=8.8 \mathrm{~Hz}, 1 \mathrm{H}, \mathrm{H}-5), 7.46 \sim 7.26(\mathrm{~m}$, $12 \mathrm{H}, \mathrm{ArH}$ ), $7.03\left(\mathrm{dd}, J=6.8,2.0 \mathrm{~Hz}, 2 \mathrm{H}, \mathrm{H}-3^{\prime}\right.$ and $\left.5^{\prime}\right), 6.68$ (d, $J=8.8 \mathrm{~Hz}, 1 \mathrm{H}, \mathrm{H}-6), 5.41$ (dd, $J=3.2,12.8 \mathrm{~Hz}, 1 \mathrm{H}$, $\mathrm{H}-2$ ), $5.23 \sim 5.20$ (m, 1H, H-2"), 5.19 (s, $2 \mathrm{H}, \mathrm{OCH}_{2}$ ), 5.10 (s, 2H, $\left.\mathrm{OCH}_{2}\right), 3.41$ (d, J=7.2 Hz, 2H, H-1"), 3.02 (dd,
$\left.J=13.2,3.6 \mathrm{~Hz}, 1 \mathrm{H}, \mathrm{H}-3_{\mathrm{ax}}\right), 2.84$ (dd, $J=16.8,3.2 \mathrm{~Hz}, 1 \mathrm{H}$, H- $3_{\text {eq }}$ ), 1.67 (d, $\left.J=1.2 \mathrm{~Hz}, 3 \mathrm{H}, \mathrm{H}-4 "\right), 1.67$ (d, $J=0.8 \mathrm{~Hz}$, $3 \mathrm{H}, \mathrm{H}-5 ") ;{ }^{13} \mathrm{C}$ NMR $\left(100 \mathrm{MHz}, \mathrm{CDCl}_{3}\right) \delta: 191.6,162.4$, $160.4,158.9,136.8,136.5,131.8,131.7,128.6,128.1$, $127.5,127.3,126.3,122.0,118.2,115.5,115.0,106.1$, $70.4,70.1,44.3,25.8,22.4,17.8$; HRMS calcd for $\mathrm{C}_{34} \mathrm{H}_{33} \mathrm{O}_{4}[\mathrm{M}+\mathrm{H}]^{+}:$: 505.2373, found 505.2371.

3.2.4 4',7-二卡氧基-8-(3,3-二甲基-2,3-环氧丙基)黄 烷酮(10)的合成

搅拌下, 将 $m$-CPBA (138 mg, $0.40 \mathrm{mmol}$ ) 的二氯甲 烷溶液 (5 mL)滴加到化合物 $9(135 \mathrm{mg}, 0.27 \mathrm{mmol})$ 的二 氯甲烷 $(5 \mathrm{~mL})$ 中, $0{ }^{\circ} \mathrm{C}$ 下摚拌 $5 \mathrm{~h}$, 加入少量水, 分离出 有机相, 水相用二氯甲烷萃取, 合并有机相, 有机相依 次用 $\mathrm{NaHCO}_{3}$, 食盐水洗, 无水硫酸钠干燥, 减压蒸去 溶剂, 硅胶柱层析分离, 得黄色油状物 $\mathbf{1 0}$ (95 mg, 0.18 mmol), 产率 68\%. ${ }^{1} \mathrm{H}$ NMR (400 MHz, $\left.\mathrm{CDCl}_{3}\right) \delta: 7.87$ (d, $J=8.8 \mathrm{~Hz}, 1 \mathrm{H}, \mathrm{H}-5), 7.46 \sim 7.32$ (m, 12H, ArH), 7.02 (dd, $J=1.2,8.8 \mathrm{~Hz}, 2 \mathrm{H}, \mathrm{H}-3$ ' and $\left.5^{\prime}\right), 6.71(\mathrm{~d}, J=8.8,1 \mathrm{H}, \mathrm{H}-6)$, $5.41(\mathrm{dd}, J=3.2,10.0 \mathrm{~Hz}, 1 \mathrm{H}, \mathrm{H}-2), 5.18(\mathrm{~s}, 2 \mathrm{H}$, $\mathrm{OCH}_{2} \mathrm{Ar}$ ), 5.10 (s, 2H, $\mathrm{OCH}_{2} \mathrm{Ar}$ ), 3.11 (dd, $J=4.4,13.6$ $\mathrm{Hz}, 1 \mathrm{H}, \mathrm{H}-2 "), 3.06 \sim 2.97$ (m, 2H, H-3 and 1"), $2.87 \sim$ 2.79 (m, 2H, H-3 and 1"), 1.22 (d, $J=4.4 \mathrm{~Hz}, 3 \mathrm{H}, \mathrm{H}-5 ")$, 1.19 (d, $J=2.8 \mathrm{~Hz}, 3 \mathrm{H}, \mathrm{H}-4 ") ;{ }^{13} \mathrm{C}$ NMR $(100 \mathrm{MHz}$, $\left.\mathrm{CDCl}_{3}\right) \delta: 191.4,162.9,160.9,159.1,136.8,136.2,131.4$, $131.2,128.7,128.3,128.1,127.8,127.5,127.4,127.2$, $115.5,115.1,114.5,106.1,79.5,740.6,70.1,63.3,59.2$, 44.5, 24.8, 22.9, 19.0; HRMS calcd for $\mathrm{C}_{34} \mathrm{H}_{32} \mathrm{O}_{5} \mathrm{Na}[\mathrm{M}+$ $\mathrm{Na}]^{+}: 543.2142$, found 543.2136 .

3.2.5 4',7-二市氧基-8-(2,3-二羟基-3-甲基丁基)黄烷 酮(11)的合成

将化合物 $\mathbf{1 0}(154 \mathrm{mg}, 0.23 \mathrm{mmol})$ 溶于 $12 \mathrm{~mL}$ THF 中, 搅拌下, 滴加 $\mathrm{H}_{2} \mathrm{SO}_{4}(49 \%) 10 \mathrm{~mL}, \mathrm{TLC}$ 监测, $5 \mathrm{~h}$ 后, 加少量水, 乙酸乙酯萃取, 合并有机相, 有机相依次食 盐水洗, 无水硫酸钠干燥, 减压蒸去溶剂, 硅胶柱层析 分离, 得黄色油状物 11 (89 mg, $0.17 \mathrm{mmol}$ ), 产率 74\%. ${ }^{1} \mathrm{H}$ NMR $\left(400 \mathrm{MHz}, \mathrm{CDCl}_{3}\right) \delta: 7.87(\mathrm{dd}, J=2.2,9.0 \mathrm{~Hz}$, H-5), $7.45 \sim 7.32(\mathrm{~m}, 12 \mathrm{H}, \mathrm{ArH}), 7.02(\mathrm{dd}, J=3.2,8.8 \mathrm{~Hz}$, $2 \mathrm{H}, \mathrm{H}-3^{\prime}$ and $\left.\mathrm{H}-5^{\prime}\right), 6.74$ (dd, $J=1.8,8.8 \mathrm{~Hz}, 1 \mathrm{H}, \mathrm{H}-6$ ), 5.42 (dd, $J=13.2,2.6 \mathrm{~Hz}, 1 \mathrm{H}, \mathrm{H}-2), 5.16$ (s, $2 \mathrm{H}, \mathrm{OCH}_{2}$ ), $5.11\left(\mathrm{~s}, 2 \mathrm{H}, \mathrm{OCH}_{2}\right), 3.59(\mathrm{t}, J=10.4 \mathrm{~Hz}, 1 \mathrm{H}, \mathrm{H}-2 "), 3.04 \sim$ $2.9\left(\mathrm{~m}, 2 \mathrm{H}, \mathrm{H}-3_{\mathrm{ax}}\right.$ and $\left.\mathrm{H}-1 "\right), 2.87 \sim 2.79\left(\mathrm{~m}, 2 \mathrm{H}, \mathrm{H}-3_{\mathrm{eq}}\right.$ and H-1"), 1.15 (s, 3H, H-4"), 1.14 (s, 3H, H-3"); ${ }^{13} \mathrm{C}$ NMR $\left(100 \mathrm{MHz}, \mathrm{CDCl}_{3}\right) \delta: 1923,162.6,160.6,159.0,136.7$, $135.9,131.0,128.8,128.7,128.4,128.1,127.5,127.4$, 127.4, 127.1, 115.6, 115.1, 79.7, 79.4, 78.8, 78.6, 72.8, 
70.8, 70.1, 44.1, 26.0, 25.8, 23.4; HRMS calcd for $\mathrm{C}_{34} \mathrm{H}_{34} \mathrm{O}_{6} \mathrm{Na}[\mathrm{M}+\mathrm{Na}]^{+}:$561.2248, found 561.2238.

\subsubsection{Brosimacutin A (1) 的合成}

化合物 11 (41 mg, $0.076 \mathrm{mmol}$ )和 $5 \mathrm{mg} 5 \% \mathrm{Pd} / \mathrm{C}$ 在 常温常压 $\mathrm{H}_{2}$ 氛围中, 以甲醇/乙酸乙酯 $(2 \mathrm{~mL} / 1 \mathrm{~mL})$ 溶剂 反应, TLC 监测, 约 $20 \mathrm{~h}$ 后, 过滤除去 $\mathrm{Pd} / \mathrm{C}$, 旋干溶剂, 硅胶柱层析分离, 得无色无定形固体 1 (23 mg, 0.064 mmol), 产率 85\%. ${ }^{1} \mathrm{H}$ NMR (400 MHz, $\left.\mathrm{CD}_{3} \mathrm{OD}\right) \delta: 7.66$ (dd, $J=8.8,2.0 \mathrm{~Hz}, 1 \mathrm{H}, \mathrm{H}-5), 7.36$ (dd, $J=8.4,1.6 \mathrm{~Hz}$, $2 \mathrm{H}, \mathrm{H}-2^{\prime}$ and $\left.6^{\prime}\right), 6.82\left(\mathrm{dd}, J=8.8,0.4 \mathrm{~Hz}, 2 \mathrm{H}, \mathrm{H}-3^{\prime}\right.$ and $5^{\prime}$ ), 5.40 (dd, $J=13.2,1.6 \mathrm{~Hz}, 1 \mathrm{H}, \mathrm{H}-2), 3.59$ (dd, $J=10.0,2.4$ $\mathrm{Hz}, 1 \mathrm{H}, \mathrm{H}-2 "), 3.05 \sim 2.97$ (m, 2H, H-3 $3_{\mathrm{ax}}$ and H-1"), 2.75 (dd, $J=8.4,2.8 \mathrm{~Hz}, 1 \mathrm{H}, \mathrm{H}-3_{\mathrm{eq}}$ ), 2.72 (dd, $J=5.6,2.8 \mathrm{~Hz}$, 1H, H-1"), 1.19 (s, 3H, H-4"), 1.17 (s, 3H, H-5"); ${ }^{13} \mathrm{C}$ NMR (100 MHz, $\left.\mathrm{CD}_{3} \mathrm{OD}\right) \delta: 194.1,164.9,163.2,158.9,131.5$, 128.9, 127.3, 116.2, 115.6, 115.2, 111.4, 80.9, 79.9, 73.9, 44.8, 26.7, 25.9, 24.8; HRMS calcd for $\mathrm{C}_{20} \mathrm{H}_{22} \mathrm{O}_{6} \mathrm{Na}[\mathrm{M}+$ $\mathrm{Na}]^{+}: 381.1309$, found 381.1305 .

\subsubsection{Brosimacutin M (2)的合成}

用 $5 \mathrm{~mL}$ 乙醇溶解 $(17 \mathrm{mg}, 0.047 \mathrm{mmol}$ ) Brosimacutin $\mathrm{A}, 0{ }^{\circ} \mathrm{C}$ 剧烈搅拌下, 滴加 $4 \mathrm{~mol} / \mathrm{L} \mathrm{KOH} 5 \mathrm{~mL}$, TLC 检测 反应, 约 $20 \mathrm{~min}$ 后, 加入少量冰水, $1 \mathrm{~mol} / \mathrm{L} \mathrm{HCl}$ 调节 $\mathrm{pH}$ 小于 3 , 乙酸乙酯萃取, 合并有机相, 依次用水、饱和食 盐水洗, 无水硫酸钠干燥, 减压蒸去溶剂, 得黄色粉末 Brosimacutin M (13 mg, $0.036 \mathrm{mmol})$ 2, 产率 77\%. ${ }^{1} \mathrm{H}$ NMR $\left(400 \mathrm{MHz}\right.$, Acetone- $\left.d_{6}\right) \delta: 8.08(\mathrm{~d}, J=8.8 \mathrm{~Hz}, 1 \mathrm{H}$, H-5), 7.86 (d, $J=15.2 \mathrm{~Hz}, 1 \mathrm{H}, \mathrm{H}-2), 7.79$ (d, $J=15.6 \mathrm{~Hz}$, $1 \mathrm{H}, \mathrm{H}-3), 7.75$ (d, $J=8.8 \mathrm{~Hz}, 2 \mathrm{H}, \mathrm{H}-2^{\prime}$ and $\left.\mathrm{H}-6^{\prime}\right), 6.93$ (d, $J=8.4 \mathrm{~Hz}, 2 \mathrm{H}, \mathrm{H}-3^{\prime}$ and $\left.\mathrm{H}-5^{\prime}\right), 6.48(\mathrm{~d}, J=8.8 \mathrm{~Hz}, 1 \mathrm{H}$, H-6), 3.63 (dd, $J=1.8,9.8 \mathrm{~Hz}, 1 \mathrm{H}, \mathrm{H}-2 "), 3.27$ (dd, $J=$ 14.0, $1.8 \mathrm{~Hz}, 1 \mathrm{H}, \mathrm{H}-1 "), 2.58$ (dd, $J=14.4,10.0 \mathrm{~Hz}, 1 \mathrm{H}$, H-1"), 1.32 (s, 3H, H-5"), 1.25 (s, 3H, H-6"); ${ }^{13} \mathrm{C}$ NMR (100 MHz, Acetone- $d_{6}$ ) $\delta: 193.0,165.2,146.6,161.0$, $145.1,131.8,130.8,127.6,118.3,116.8,114.9,114.2$, 109.3, 80.8, 72.8, 25.9, 24.7, 26.0; HRMS calcd for $\mathrm{C}_{20} \mathrm{H}_{22} \mathrm{O}_{6} \mathrm{Na}[\mathrm{M}+\mathrm{Na}]^{+}: 381.1309$, found 381.1036.

\subsubsection{Brosimacutin $\mathrm{H} \mathrm{(3)}$ 的合成}

化合物 $2(12 \mathrm{mg}, 0.034 \mathrm{mmol}$ ) 和 $2 \mathrm{mg} 5 \% \mathrm{Pd} / \mathrm{C}$ 在常 温常压 $\mathrm{H}_{2}$ 氛围中, 以甲醇/乙酸乙酯 $(1 \mathrm{~mL} / 0.5 \mathrm{~mL})$ 为溶 剂反应, TLC 监测, 约 $10 \mathrm{~h}$ 后, 过滤除去 $\mathrm{Pd} / \mathrm{C}$, 旋干溶 剂, 硅胶柱层析分离, 得无色无定形固体 3 ( $2 \mathrm{mg}, 0.017$ mmol), 产率 50\%. ${ }^{1} \mathrm{H} \mathrm{NMR}\left(400 \mathrm{MHz}, \mathrm{CD}_{3} \mathrm{OD}\right) \delta: 7.66$ (dd, $J=8.8,2.0 \mathrm{~Hz}, 1 \mathrm{H}, \mathrm{H}-5), 7.05$ (d, $J=8.8 \mathrm{~Hz}, 2 \mathrm{H}$, H-2'), 6.69 (dd, $\left.J=6.4,2.0 \mathrm{~Hz}, 2 \mathrm{H}, \mathrm{H}-3^{\prime}\right), 6.40$ (d, $J=9.2$ $\mathrm{Hz}, 1 \mathrm{H}, \mathrm{H}-6), 3.59$ (dd, $J=10.0,2.4 \mathrm{~Hz}, 1 \mathrm{H}, \mathrm{H}-2 "), 3.19$ (t,
$J=7.6 \mathrm{~Hz}, 2 \mathrm{H}, \mathrm{H}-3$ ), 3.05 (dd, $J=13.6,2.4 \mathrm{~Hz}, 1 \mathrm{H}, \mathrm{H}-1 ")$, 2.91 (t, $J=7.6 \mathrm{~Hz}, 2 \mathrm{H}, \mathrm{H}-2), 2.70$ (dd, $J=14.0,10.0 \mathrm{~Hz}$, $1 \mathrm{H}, \mathrm{H}-1 "), 1.25$ (s, 6H, H-4" and $5 ") ;{ }^{13} \mathrm{C}$ NMR (100 MHz, $\left.\mathrm{CD}_{3} \mathrm{OD}\right) \delta: 205.9,164.5,164,156.7,133.2,131.3,130.4$, 116.2, 114.9, 113.9, 108.8, 79.9, 74.0, 40.9, 31.1, 26.0, 25.6, 25.2; HRMS calcd for $\mathrm{C}_{24} \mathrm{H}_{24} \mathrm{O}_{6} \mathrm{Na}[\mathrm{M}+\mathrm{Na}]^{+}$: 383.1465, found 383.1461.

\subsubsection{Brosimacutin E (5)的合成}

将化合物 $10(65 \mathrm{mg}, 0.13 \mathrm{mmol})$ 溶于 $2 \mathrm{~mL}$ 乙醇, $\mathrm{Pd}^{-} \mathrm{CaCO}_{3}(260 \mathrm{mg}) \mathrm{N}_{2}$ 环境下将 1,4-环已二烯 $(416 \mathrm{mg}$, $5.2 \mathrm{mmol}$ )滴加到上述溶液中, TLC 监测, $40{ }^{\circ} \mathrm{C}$ 下反应 24 $\mathrm{h}$, 反应结束后, 过滤除去 $\mathrm{Pd}-\mathrm{CaCO}_{3}$, 旋干溶剂, 硅胶 柱层析分离, 得无色无定形固体 $5(34 \mathrm{mg}, 0.10 \mathrm{mmol})$, 产率 83\%. ${ }^{1} \mathrm{H}$ NMR $\left(400 \mathrm{MHz}, \mathrm{CD}_{3} \mathrm{OD}\right) \delta: 7.71(\mathrm{~d}, J=7.2$ Hz, 1H, H-5), 7.30 (dd, $J=8.4,2.4 \mathrm{~Hz}, 2 \mathrm{H}, \mathrm{H}-2^{\prime}$ ), 6.81 (dd, $J=8.4,2.0 \mathrm{~Hz}, 2 \mathrm{H}, \mathrm{H}-3$ '), 6.49 (d, $J=8.8 \mathrm{~Hz}, 1 \mathrm{H}$, H-6), 5.37 (dd, $J=12.4,2.8 \mathrm{~Hz}, 1 \mathrm{H}, \mathrm{H}-2$ ), 4.70 (t, $J=9.0$ Hz, 1H, H-2"), 3.09 (d, $J=9.6 \mathrm{~Hz}, 2 \mathrm{H}, \mathrm{H}-1$ "), 3.02 (dd, $\left.J=15.8,12.6 \mathrm{~Hz}, 1 \mathrm{H}, \mathrm{H}-3_{\mathrm{ax}}\right), 2.67$ (dd, $J=14.6,2.2 \mathrm{~Hz}$, $1 \mathrm{H}, \mathrm{H}-3_{\mathrm{eq}}$ ), 1.26 (s, 3H, H-5"), 1.19 (s, 3H, H-4"); ${ }^{13} \mathrm{C}$ NMR (100 MHz, $\left.\mathrm{CD}_{3} \mathrm{OD}\right) \delta: 193.3,168.9,160.3,160.0$, 131.1, 129.8, 129.1, 116.4, 116.2, 115.2, 105.3, 92.7, 81.0, 72.3, 44.8, 28.1, 25.4, 25.2; HRMS calcd for $\mathrm{C}_{20} \mathrm{H}_{20} \mathrm{O}_{5} \mathrm{Na}$ $[\mathrm{M}+\mathrm{Na}]^{+}: 363.1203$, found 363.1198 .

\subsubsection{Brosimacutin D (4) 的合成}

将化合物 $12(61 \mathrm{mg}, 0.12 \mathrm{mmol})$ 和 Pd-C (6 mg)溶于 $3 \mathrm{~mL}$ 乙酸乙酯中, 氢气氛围中反应 $4 \mathrm{~d}$ 后, 加入 $0.2 \mathrm{~mL}$ 甲酸, 继续反应 $2 \mathrm{~h}$, 过滤除去 $\mathrm{Pd} / \mathrm{C}$, 旋干溶剂, 硅胶柱 层析分离, 得无色无定形固体 4 (14 mg, $0.04 \mathrm{mmol})$, 产 率 33\%. ${ }^{1} \mathrm{H}$ NMR $\left(400 \mathrm{MHz}, \mathrm{CD}_{3} \mathrm{OD}\right) \delta: 7.64(\mathrm{~d}, J=8.8$ Hz, 1H, H-5), 7.35 (d, $J=8.4$ Hz, 2H, H-2'), 6.83 (d, $J=$ $8.4 \mathrm{~Hz}, 2 \mathrm{H}, \mathrm{H}-3$ '), 6.48 (d, $J=8.8 \mathrm{~Hz}, 1 \mathrm{H}, \mathrm{H}-6), 5.42$ (dd, $J=13.2,2.4 \mathrm{~Hz}, 1 \mathrm{H}, \mathrm{H}-2), 3.76(\mathrm{dd}, J=10.6,5.0 \mathrm{~Hz}, 1 \mathrm{H}$, H-2"), 3.05 (dd, $\left.J=16.8,13.2 \mathrm{~Hz}, 1 \mathrm{H}, \mathrm{H}-3_{\mathrm{ax}}\right), 2.91$ (dd, $J=17.2,5.2 \mathrm{~Hz}, 1 \mathrm{H}, \mathrm{H}-1 "), 2.73$ (dd, $J=16.8,2.8 \mathrm{~Hz}, 1 \mathrm{H}$, H-3 ${ }_{\text {eq }}$ ), 2.57 (dd, $\left.J=17.2,7.2 \mathrm{~Hz}, 1 \mathrm{H}, \mathrm{H}-1 "\right), 1.32$ (s, 3H, H-5"), 1.31 (s, 3H, H-4"); ${ }^{13} \mathrm{C}$ NMR (100 MHz, $\left.\mathrm{CD}_{3} \mathrm{OD}\right) \delta$ : $193.8,163.0,161.4,159.0,131.3,128.9,116.3,115.0$, 112.6, 109.5, 81.1, 79.4, 69.5, 44.7, 26.7, 25.5; HRMS calcd for $\mathrm{C}_{20} \mathrm{H}_{20} \mathrm{O}_{5} \mathrm{Na}[\mathrm{M}+\mathrm{Na}]^{+}:$363.1203, found 363.1200 .

\section{References}

[1] Abeza, B. M.; NgadJui, B. T.; Dongo, E.; Ngameni, B.; Nindi, M. N.; Bezabih, M. Phytochemistry 2002, 59, 877.

[2] Cidade, H. M.; Nacimento, M. S.; Pinto, M. M. M.; KiJJoa, A.; 
Silva, A. M. S.; Herz, W. Planta Med. 2001, 67, 867.

[3] Meragelman, K. M.; Mckee, T. C.; Boyd, M. R. J. Nat. Prod. 2001, $64,546$.

[4] Dufall, K. G.; NgadJui, B. T.; Simeon, K. F.; Abegaz, B. M.; Abegaz, K. D.; Croft, K. D. J. Ethnopharmacol. 2003, 87, 67.

[5] Barron, D.; Ibraham, R. K. Phytochemistry 1996, 43, 921.

[6] Tadigoppula, N.; Korthikunta, V.; Gupta, S.; Kancharla, P.; Khaliq, T.; Soni, A. J. Med. Chem. 2013, 56, 31.

[7] TaKashima, J.; Ohsaki, A. J. Nat. Prod. 2001, 64, 1493.

[8] TaKashima, J.; Ohsaki, A. J. Nat. Prod. 2002, 65, 1483.

[9] TaKashima, J.; Ohsaki, A. Planta Med. 2005, 71, 654.

[10] Yang, J. H.; Zuo, W. B.; Guo, D. D.; Luo, J. S.; Huang, W. Q. Chin. Chem. Lett. 2012, 23, 1375.

[11] Yang, J. H.; Huang, W. Q.; Luo, J. S.; Guo, D. D.; Zhang, Y. H.; Li, H. J. Chin. Chem. Lett. 2012, 23, 127.

[12] Yang, J. H.; Chen, B. B.; Xie, Y. M.; Feng, Y.; Ma, X. Q. Chin. Chem. Lett. 2013, 24, 1027.
[13] Yang, J. H.; Xie, Y. M.; Feng, S. B.; Zuo, W. B.; Cheng, B. B.; Ma, X. Q.; Feng, Y. Chin. J. Org. Chem. 2012, 32, 2159 (in Chinese). (杨金会, 谢一民, 冯尚彪, 左武标, 陈兵兵, 马晓琴, 冯尧, 有 机化学, 2012, 32, 2159.)

[14] Jagannadha, R. N.; Narendra, K. M.; Murali, M.; Satyanarayana, P. V. V. Asian J. Chem. 2007, 19, 2885.

[15] Kaur, N.; Xia, Yan.; Jin, Y.; Dat, N. T.; GaJulapati, K.; Choi, Y.; Hong, Y.; Lee, J. J.; Lee, K. Chem. Commun. 2009, 1879.

[16] Xie, Z. X.; Hu, Y.; Li, Y. J. Chin. Pharm. Sci. 2004, 13, 115 (in Chinese). (谢志翔, 胡杨, 李泫, 中国药学, 2004, 13, 115.)

[17] Nagayama, S.; Endo, M.; Kobayashi, S. J. Org. Chem. 1998, 18, 6094.

[18] Rani, S.; Vankar, Y. D. Tetrahedron Lett. 2003, 907.

[19] Satoshi, T., John, L. I., Junya, M. Phytochemistry 1989. $28,2079$.

[20] Jun, N.; Hong, G.; Jun, K. Bioorg. Med. Chem. 2007, 15, 2396.

(Li, L.; Lu, Z.) 